\title{
Towards efficient ftight: insights on proper morphing-wing modulation in a bat-like robot
}

\author{
J. Colorado, C. Rossi, C. Zhang and A. Barrientos
}

\begin{abstract}
In this article, we address the question of how the ftight efficiency of Micro Aerial Vehicles with variable wing geometry can be inspired by the biomechanics of bats. We use a bat-Iike drone with highly articulated wings using shape memory alloys (SMA) as artificial muscle-Iike actuators. The possibility of actively changing the wing shape by controlling the SMA actuators, Jet us study the effects of different wing modulation pattems on lift generation, drag reduction, and the energy cost of a wingbeat cycle.To this purpose, we presentan energy-model for estimating the energy cost required by the wings during a wingbeat cycle, using experimental aerodynamic and inertial force data as inputs to the energy-model. Results allowed us determining that faster contraction of the wings during the upstroke, and slower extension during the downstroke enables to reduce the energy cost of ftapping in our prototype.
\end{abstract}

Keywords:morphing-wings; bat ftight; shape memory alloys

\section{Introduction}

Micro Aerial Vehicles (MAV) are a class of unmanned aerial vehicles whose size is usually of the order of few centimeters of wingspan and mass less than few hundred grams (cf. Figure 1). Thanks to their small size and high maneuverability, MAVs are ideal for many applications such as reconnaissance in confined spaces, search and rescue, or hazardous environment exploration. Because of their size, however, the design of MAVs implies important technological challenges, such as, maniobrability and low power consumption.

Recently, roboticists have started to demonstrate a special interest in learning from nature as the key to optimize flight efficiency.[1-4] The concept of morphing-wing Micro Aerial Vehicles comes from nature.[5,6] Unlike insects and birds, bat wings are provided with a complex musculature and articulations that enable a high degree of dexterity.[7,8] This allows bats to save more energy during flight than any other flying creature.[9-12] Also, their massive wings undergo large accelerations that are caused by inertial forces with a significan!contribution for maneuvering.[13]

One of the first works that attempted to reproduce bioinspired bat flight using SMA was presented in [14] and [15]. Later in [16,17], it was carried out a study of different materials for the wing membrane: $n$ ylon, spandex, and si licone. Aerodynamic measurements demonstrated that silicone-made membrane achieved higher lift-to-drag ratios. Recentiy, in [18] it was proposed the design of a highly articulated wing structure inspired by the morphology of the Cynopterus brachiotus bat speci men. They chose that species because its well-studied in-vivo wing kinematics and aerodynamics. Results from [18] report experimental measurements that detail the inertial and aerodynamic power involved in the cost of flapping and the contribution of the wing inertia in the overall cost of flight.

In this article, we useBaTboT, a bat-like drone inspired by the articulated wing design from Bahlman et al. [18], and originally developed with the collaboration of Breuer et al. [19]. BaTboT has highly articulated wings using shape memory alloys (SMA) as artificial muscle-like actuators. Due to size and weight limitations, classical servo-motor technology may not be the most suitable actuation technology for the purpose of morphing-wing control. In fact, most of the prototypes designed in this field adopt altemative actuation technology based onfunctional (or smart) materials.

Based on the possibility of actively changing BaTboTs wing shape by controlling the SMA actuators, here we introduce an energy-cost modeling framework for providing insights on the energy consu mption of the robot during a wingbeat cycle. We aim to study how different wing modulation patterns (wing extension/retraction) affect the energy consumption, lift, drag, and net body force production. As a result, we expect to find the most suitable wing modulation pattem that enables our robot to generate more lift, thrust, and reduce drag at the expense of acceptable energy consumption. 


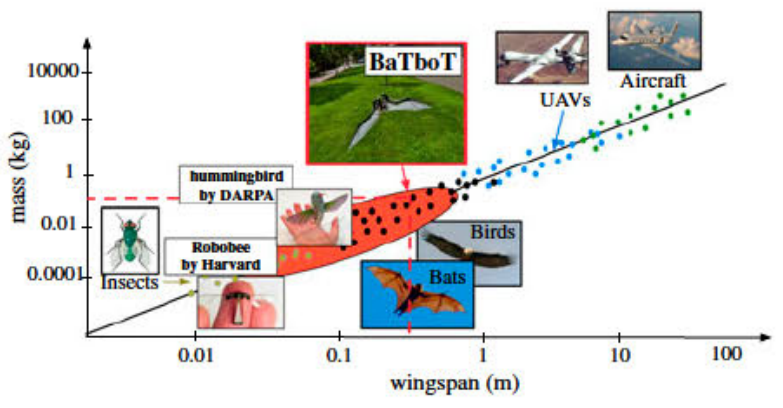

Figure 1. MAVs fill the niche of $1-50 \mathrm{~cm}$ wingspan and about $1-100 \mathrm{~g}$ weight.

\section{The BaTboT}

Starting from Bahlman's robotic wing design [18] and with the collaboration of Breuer et al. [19] we have developed the robotic model illustrated in Figure 2, called BaTboT. Our work is motivated by the potential behind bat flight and by the lack of highly articulated morphing-wing aerial drones.

The skeletal structure of BaTboT wings can be actuated by Shape Memory Alloy acting as artificial triceps and biceps muscles. BaTboT has an overall mass of $125 \mathrm{~g}$ (including battery) and is composed by two highly articulated wings $(9 \mathrm{~g})$ attached to a mainframe body $(38 \mathrm{~g})$. Each wing of the robot has six degrees of freedom (DoF): two DoF at the shoulder driven by a DC-motor, one DoF at the elbow and three $\mathrm{DoF}$ at the wrist joint, both driven by Nickel Titanium (NiTi) shape memory alloy musclelike actuators supplied by Migamotors. These muscles enableBaTboT to change its wingspan from $40.8 \mathrm{~cm}$ (wings retracted) to $53 \mathrm{~cm}$ (wings fully extended). The SMA artificial muscles are essential to provide mechanical simplicity and lightweight to the morphing wing mechanism. Each SMA actuator has a mass of $1.1 \mathrm{~g}$.

In previous work [19], we carried out experiments to determine how to increase the SMA actuation bandwidth and the effects of SMA fatigue issues depending on the applied power. Also in [20], we studied alternatives for flight control by developing an inertial-model for estimating the spatial forces produced by both massive wings and its role for manoeuvring, i.e roll and pitch motions of the robot. In this paper, we introduce an energy-cost modelling framework that allows for the estimation of the energy consumption for different wing kinematics, something that is extremely difficult to perform in animals. Our work can provide insights on:

(1) Designing highly articulated morphing wings driven by SMA actuators.

(2) Characterizing SMA actuation and control.

(3) Increasing flight performance via proper wing modulation.
Appendix 1 briefly details how to control the morphing wings using the SMA actuators based on previous work [19].

\section{Energy-cost modelling framework}

The kinetic energy for both wings during a wingbeat cycle is a contribution given by the inertia of composite-rigid-body wing skeleton and the joint velocities. Likewise, the kinetic energy changes depending on the wingbeat phase: downstroke or upstroke. To estimate the overall energy cost of the flapping and morphing-wing motions and their impact onto the robot's center of mass, a rigid-body dynamic model for $B a T b o T$ is required. This model calculates the spatial inertia tensor or inertia matrix that defines the relationship between BaTboT body velocity and momentum. The term spatial refers to the use of six-dimensional (6D) vectors in which the linear and angular components of rigid-body motion are combined into a unified set of physical quantities.[21,22] Hence, the inertia matrix $I_{b}$ with respect to the body frame $\{b\}$ (see Figure 3 ) is estimated by the sum of the inertias of all the rigid bodies that compose both wings. Note in Figure 3 that each wing of $B a T b o T$ is kinematically represented as a serial chain of articulated rigid bodies $i$ connected to a floating base (body frame $\{b\}$ ).

In this paper, we use the measurements of lift, drag, and thrust captured during wind tunnel experiments carried out in [20] to provide a real input of the inertial and aerodynamic forces to the energy model. Based on that, we first calculate the spatial inertia $\left(I_{b}\right)$ acceleration $\left(\dot{V}_{b}\right)$ and velocity $\left(V_{b}\right)$ of $B a T b o T$ body to finally estimate the overall energy cost $(K)$. The subscript $b$ indicates that the physical quantity is with respect to the body frame.

\subsection{Inertia of the composite-rigid-body articulated wings}

Each rigid-body $i$ of the wing structure (see Figure 3) has a $6 \times 6$ inertia matrix corresponding to $I_{i}=\hat{S}_{i, c m} I_{c m} \hat{S}_{i, c m}^{T}$, as shown in (1). The subscripts $\{i\}$ and $\{\mathrm{cm}\}$ indicate whether the corresponding physical quantity is with respect the joint frame or the center of mass frame, respectively. The expression $\hat{S}_{i, c m} I_{c m} \hat{S}_{i, c m}^{T}$ refers to the translation of the inertia matrix calculated with respect to the center of mass frame $\left(I_{c m}\right)$ by applying the parallel axis theorem. In this sense, the term $\hat{S}_{i, c m}$ is a $6 \times 6$ matrix formed by the $3 \times 3$ skew symmetric matrix $\tilde{s}_{i, c m}$ of the position vector $\vec{s}_{i, c m}$ that connects the joint frame $\{i\}$ with the center of mass frame $\{\mathrm{cm}\}$ of the rigid body $i$, as shown in Figure 3 .

$$
\begin{aligned}
& I_{i}=\hat{S}_{i, c m} I_{c m} \hat{S}_{i, c m}^{T} \\
& =\left[\begin{array}{cc}
U & \tilde{s}_{i, c m} \\
0 & U
\end{array}\right]\left[\begin{array}{cc}
J_{i, c m} & 0 \\
0 & m_{i} U
\end{array}\right]\left[\begin{array}{cc}
U & 0 \\
-\tilde{s}_{i, c m} & U
\end{array}\right] \\
& =\left[\begin{array}{cc}
J_{i} & m_{i} \tilde{s}_{i, c m} \\
-m_{i} \tilde{s}_{i, c m} & m_{i} U
\end{array}\right]
\end{aligned}
$$




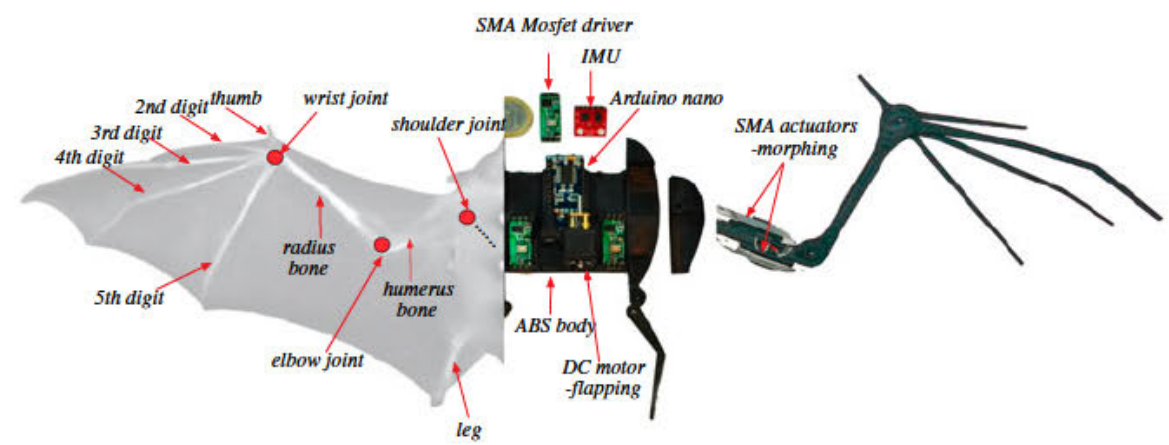

Figure 2. The BaTboT: a highly articulated bat-like drone based on Bahlman et al., robotic wing design,[18] but driven by shape memory alloy artificial muscles.[19]

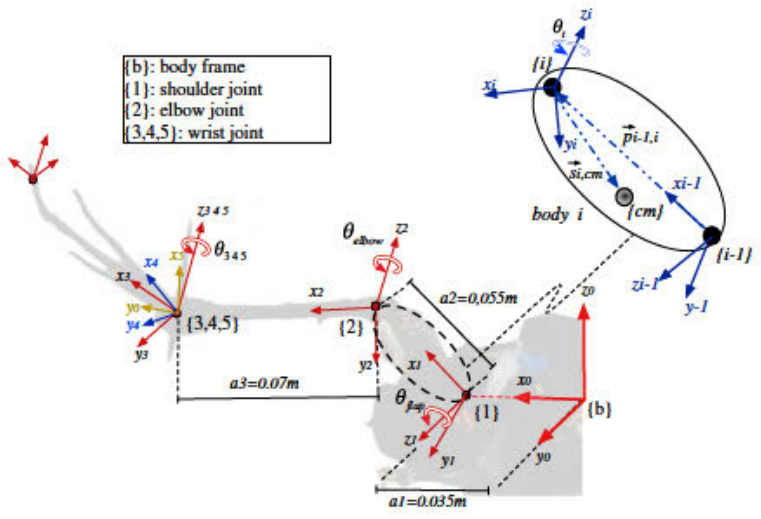

Figure 3. Wing kinematics: frames of reference based on the DH parameters.[23]

In (1), $J_{i, c m}$ is the $3 \times 3$ inertia tensor that contains the moments and products of inertia of the rigid body $i$ with mass $m_{i}$, and the term $U$ corresponds to the identity operator. In order to propagate the inertias of the wings onto the body frame $\{b\}$, spatial operators for translation $\left(\hat{P}_{i-1, i}\right)$ and rotation $\left(\hat{R}_{i, i-1}\right)$ are required. The operators described in (2) are $6 \times 6$ matrixes that depend on the wing kinematics. The term $\tilde{p}_{i-1, i}$ is the $3 \times 3$ skew symmetric matrix of the position vector $\vec{p}_{i-1, i}$ that connects the joint frame $\{i-1\}$ with the frame $\{i\}$ as shown in Figure 3. The term $r_{i, i-1}$ is the $3 \times 3$ basic rotation matrix that relates the orientation of frame $\{i\}$ with respect to the previous frame in the serial chain $\{i-1\}$. The wing frames detailed in Figure 3 are placed based on the Denavit-Hartenberg (DH) parameters.[23]

$$
\hat{P}_{i-1, i}=\left[\begin{array}{cc}
U & \tilde{p}_{i-1, i} \\
0 & U
\end{array}\right], \hat{R}_{i, i-1}=\left[\begin{array}{cc}
r_{i, i-1} & 0 \\
0 & r_{i, i-1}
\end{array}\right]
$$

Using the spatial operators in (2), the inertia matrix $I_{b}$ in (3) can be calculated by propagating the inertia of both wings using the parallel axis theorem, where $I_{o}$ is the inertia of the robot's body and $I_{i}$ is the propagated inertias of the composite-rigid-body articulated wing skeleton.

$$
\begin{aligned}
I_{b}= & I_{o}+\hat{R}_{1,0} \\
& {\left[\sum_{i=n}^{1}\left(\hat{R}_{i, i-1} P_{i-1, i}^{T}\right) I_{i}\left(R_{i, i-1} P_{i-1, i}^{T}\right)^{T}\right] }
\end{aligned}
$$

\subsection{Spatial velocities, accelerations and forces}

Spatial velocities, accelerations and forces acting on the robot's body are denoted based on the Newton-Euler formalism using spatial operators.[21,22] As previously mentioned, spatial operators lead to six-dimensional physical quantities with both angular and linear motions combined into a single operator as:

$$
V_{b}=\left[\begin{array}{c}
\omega_{b} \\
v_{b}
\end{array}\right], \dot{V}_{b}=\left[\begin{array}{c}
\dot{\omega}_{b} \\
\dot{v}_{b}
\end{array}\right], F_{b}=\left[\begin{array}{c}
\tau_{b} \\
f_{b}
\end{array}\right] \in \Re^{6 x 1}
$$

The terms $\omega_{b}$ and $v_{b}$ are the corresponding angular and linear velocities of the rigid body $i$ and $\tau_{b}, f_{b}$ the torques and forces acting on that body. Here, the spatial forces $F_{b}$ have been experimentally measured, as shown in Figure 4 . $B a T b o T$ is mounted on top of a $6 \mathrm{D}$ force sensor from which the components of $F_{b}$ are quantified [20] with respect to the body frame $\{b\}$ as:

$$
F_{b}=\left[\begin{array}{llllll}
\tau_{x 0} & \tau_{y 0} & \tau_{z 0} & f_{x 0} & f_{y 0} & f_{z 0}
\end{array}\right]^{T}
$$

In (5), the components of $F_{b}$ are the thrust $f_{T}=f_{y 0}$, the weight of the robot $f_{m g}=-f_{z 0}$, the vertical lift force component $f_{L}=f_{z 0}$ and the horizontal drag force component $f_{D}=-f_{y 0}$. The net lift $L$ and drag $D$ are calculated in (6) as a function of the angle of attack $\alpha$, as shown in Figure 5.

$$
\begin{aligned}
& L=f_{L} \cos (\alpha)-f_{D} \sin (\alpha) \\
& D=f_{D} \cos (\alpha)+f_{L} \sin (\alpha)
\end{aligned}
$$

Finally, the net body force component $F_{\text {net }}$ (see Figure 5) is quantified as:

$$
F_{n e t}=\left(L-f_{m g}\right)+\left(f_{T}-D\right)
$$




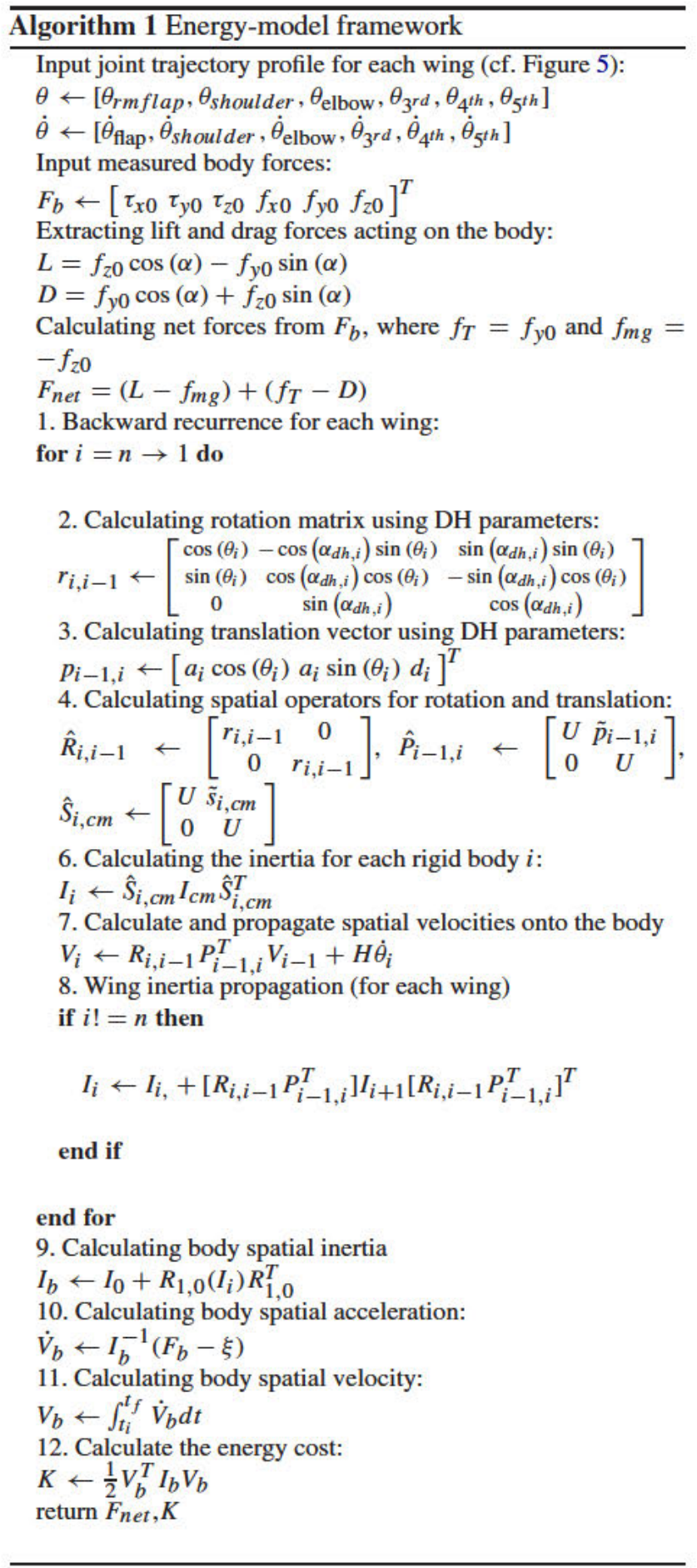

\subsection{Kinetic energy}

The kinetic energy of the system can be easily calculated as the follows:

$$
K=\frac{1}{2} V_{b}^{T} I_{b} V_{b},
$$

where $I_{b} \in \mathfrak{R}^{6 x 6}$ is the inertia matrix of the system calculated in (3) and $V_{b}$ is the spatial velocity of BaTboT body calculated as:

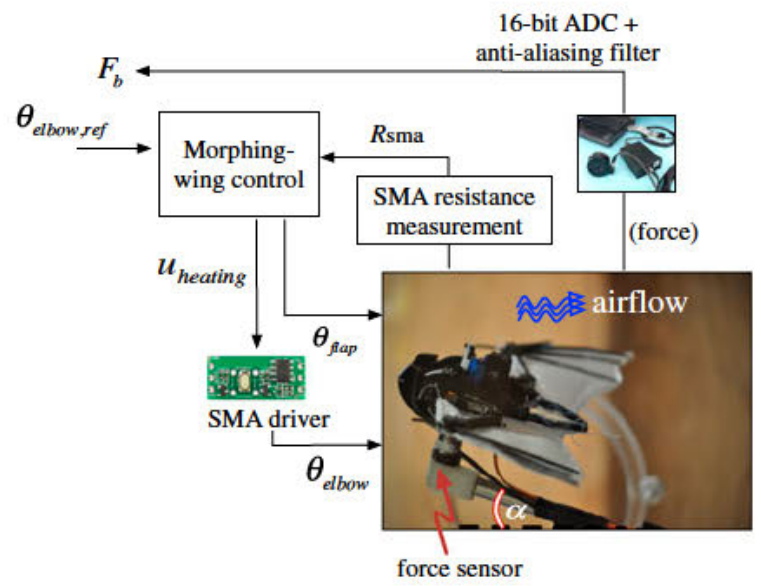

Figure 4. Experiment setup. The robot is mounted on top of a $6 \mathrm{D}$ force sensor from which the components of $F_{b}$ are measured, cf. (5). Both aerodynamic and inertial force data is then used as inputs to the energy-model proposed in this work.

$$
\begin{aligned}
F_{b} & =I_{b} \dot{V}_{b}+\xi, \\
\dot{V}_{b} & =I_{b}^{-1}\left(F_{b}-\xi\right), \\
V_{b} & =\int \dot{V}_{b} d t
\end{aligned}
$$

The term $\xi$ is the non-linear velocity-dependent force component vector, which is calculated as follows:

$$
\begin{aligned}
& \xi=\sum_{i=n}^{1}\left(\left[I_{i} \hat{S}_{i, c m}+\dot{I}_{i}\right] V_{i}\right) \\
& V_{i}=\sum_{i=n}^{1}(\underbrace{R_{i, i-1} P_{i-1, i}^{T} V_{i-1}}_{\text {propagated }}+\underbrace{H \dot{\theta}_{i}}_{\text {local }}) .
\end{aligned}
$$

In (10), the term $V_{i}$ corresponds to the spatial velocity of a rigid body $i$ and it is calculated by: (i) projecting the angular speed of the joint $i$ (denoted by $\dot{\theta}_{i}$ ) onto the corresponding axis of motion: $H \dot{\theta}_{i}$ being $H=\left[\begin{array}{llllll}0 & 0 & 1 & 0 & 0 & 0\end{array}\right]^{T}$ and (ii) propagating the spatial velocity effects from the wings to the body: $R_{i, i-1}^{T} P_{i-1, i} V_{i-1}$. The energy cost model has the advantage of using spatial operators (6D vectors) that contain both rotational and translation physical effects into a single quantity. This enables to easily operate aerodynamic forces (lift, drag) with inertial forces (weight, thrust), as shown in (5). More importantly, $I_{b} \in \Re^{6 x 6}$ has been calculated as the Spatial-Inertia of both wings,[21] which enables to define the relationship between velocity and momentum. Spatial inertia is therefore a mapping from $M^{6}$ to $F^{6}$, being $M^{n}$ spatial motion vectors and $F^{n}$ spatial force vectors. This mapping is key in our model because the components of $F_{b}$ in (9) are spatial motion vectors. Also, the inertia of a rigid body that is composed of multiple parts is the sum of the inertias of its parts. Essentially, $I_{b}$ contains the inertial information of a 12 degree of freedom wings structure. The Algorithm 1 details the procedure to compute the energy model. 


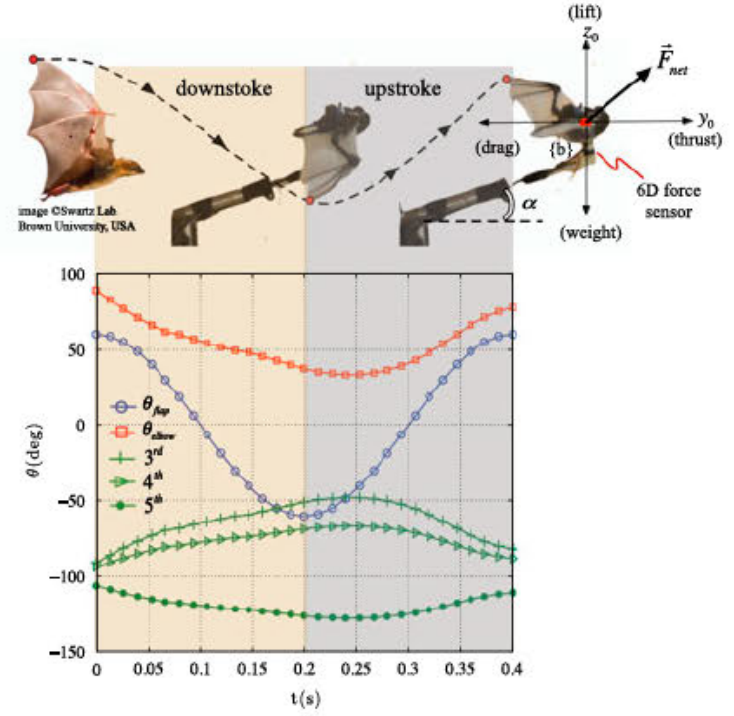

Figure 5. Wing modulation kinematics: pattern of motion described by the wing joints at a wingbeat frequency of $f_{\text {flap }}=$ $2.5 \mathrm{~Hz}$.

\section{Experimental measurements}

Experiments show the impact of applying different wing modulation patterns on:

(1) lift, drag and net body force generation, and

(2) energy cost. An interesting aspect of (8) relies on its indirect dependency on the input forces $F_{b}$, since it has been experimentally measured for different profiles of flapping frequencies $\left(f_{\text {flap }}\right)$, airflow velocities $\left(V_{\text {air }}\right)$ and angle of attacks $(\alpha)$. The energymodel framework described in Algorithm 1 can be used for analyzing the energy needed to overcome aerodynamic loads mostly during the upstroke phase of the wingbeat cycle.

In the forthcoming experiments, wing modulation patterns refer to the trajectory followed by the elbow join (contraction/extension) during a wingbeat cycle. Figure 4 describes the experimental setup. Using the morphing-wing control module (see more details on Appendix 1), we are able to generate the wing joint trajectories shown in Figure 5 . In the following experiments, we have set the primary flapping motion $\left(\theta_{\text {flap }}\right)$ with a constant wingstroke amplitude of $120^{\circ}$. Therefore, our goal here is to actively change the wing contraction and extension profile by controlling the SMA actuators that drive the elbow joint $\left(\theta_{\text {elbow }}\right)$.

The robot features a hybrid actuation system, partially actuated by a DC-motor which drives the primary flapping motion $\left(\theta_{\text {flap }}\right)$, and SMA actuators for the morphing-wing motion $\left(\theta_{\text {elbow }}\right)$. Both control signals are independent, as shown in Figure A2. The flapping motion can be increased up to $10 \mathrm{~Hz}$, nonetheless, the SMA actuation speed limits the morphing-wing motion to $2.5 \mathrm{~Hz}$. Therefore, both flapping and morphing cycles can be synchronized at a wingbeat frequency between 2 and $2.5 \mathrm{~Hz}$ due to the limitations in SMA actuation speed. Other limitations of our current robot are listed as follows:

- The robot is tethered and mounted on top of a force sensor. Therefore, it is not possible to directly analyze the effects that aerodynamic and inertial forces have onto the $6 \mathrm{D}$ motion of the robot: rotation and translation. However, we do have the $6 \mathrm{D}$ forces acting on the robot's center of mass. Using an inertialmodel developed in [20], we are able to estimate the rotations of the robot, i.e. roll, pitch and yaw, and even predict how the robot would behave in free-flight for both forward and turning maneuvers.

- In our morphing-wing control scheme (see Appendix $1)$, the electrical resistance of the SMA $\left(R_{s m a}\right)$ is the only measured variable, and the controller is servoing the electrical resistance to follow the commanded profile $\theta_{\text {elbow }}$. We know that $R_{s m a}$ changes linearly with the SMA strain that is kinematically coupled to the rotation of the elbow joint (more details on [19]), however, we do not have a direct feedback of the angle position to ensure the tracking error tends to zero.

- SMAfatigue issues: as an attempt to increase control bandwidth, SMA are constantly subject to higher stresses and larger input electrical currents, causing a critical reduction of the nominal contraction speed after several minutes of SMA continuous operation. In previous work [19], we quantified the SMA actuation speed under nominal $(1.36 \mathrm{~W})$ and overloaded $(3.06 \mathrm{~W})$ input powers. On average, we measured that after 1.5 minutes of SMA continuous operation, the morphing actuation frequency is reduced by $30 \%$. However, once the SMA actuators are completely cooled, they are able to raise the maximum operating frequency $(2.5 \mathrm{~Hz})$ for another cycle.

- The silicone-based wing membrane lacks the mechanical and morphological properties of the biological counterpart. Bat's membranes are highly anisotropy, they have tiny embedded muscles and even tiny hairs that sense airflow conditions [24].

Following the original wing design from Bahlman's et al. [18], the digits attached to the wrist joint are underactuated, which means their motion is linked to the elbow actuation via steel tendons that connect the elbow with wrist joint. Therefore, the morphing-wing motion is described by the elbow joint angle $\theta_{\text {elbow }}$ and the wrist joint angles $\theta_{3^{r}}, \theta_{4^{t h}}, \theta_{5^{t h}}$. These angles correspond to the digit III, IV, and V, respectively. The applied kinematic pattern in Figure 5 shows how during the downstroke phase both wings extend to increase the lift surface, whereas during the upstroke both wings retract to reduce drag, or aerodynamic 
Table 1. BaTboT's morphological and modelling parameters.

\begin{tabular}{lcc}
\hline Parameter & Symbol (unit) & Robot \\
\hline Body mass (with battery) & $M_{b}(\mathrm{~g})$ & 125 \\
Wings mass (with membrane) & $m_{w}(\mathrm{~g})$ & 46.15 \\
Wing membrane thickness & $(\mathrm{m})$ & 0.0001 \\
Extended wing length & $B(\mathrm{~m})$ & 0.245 \\
Body width & $l_{m}(\mathrm{~m})$ & 0.04 \\
Body moments of inertia & {$\left[I_{b, x x}, I_{b, y y}, I_{b, z z}\right]\left(\mathrm{gcm}^{2}\right)$} & {$[1,0.07,0]$} \\
Extended wing span & $S=l_{m}+2 B(\mathrm{~m})$ & 0.53 \\
Extended wing area & $A_{b}\left(\mathrm{~m}^{2}\right)$ & 0.05 \\
Humerus length & $l_{h}(\mathrm{~m})$ & 0.055 \\
Humerus moments of inertia & {$\left[I_{2, x x}, I_{2, y y}, I_{2, z z}\right]\left[\mathrm{gcm}^{2}\right]$} & {$[0.03,0.37,0.93]$} \\
Radius length & {$\left[I_{3, x x}, I_{3, y y}, I_{3, z z}\right]\left(\mathrm{gcm}^{2}\right)$} & 0.070 \\
Radius moments of inertia & & {$[0.07,0.92,0.37]$} \\
\hline
\end{tabular}

friction. During wing contraction and extension, the lifting area varies from 0.0385 to $0.05 \mathrm{~m}^{2}$. Table 1 describes the numerical values for modelling parameters.

The column $(a)$ of Figure 6 summarizes the results of applying three different wing modulation patterns (elbow joint profile $\left.\theta_{\text {elbow }}\right)$. Such profiles differ in the proportion that the wing takes for contraction and extension during the wingbeat cycle. The wing modulation patterns are not periodic due to the behavior of the SMA actuators. First of all, the elbow position is indirectly calculated by measuring the changes on SMA wire electrical resistance: it changes almost as a linear function of the angular position of the elbow joint [25]. One might expect that the resistance-motion relationship provides a feasible measurement of the elbow angle at any condition, but instead, accumulative errors are introduced during the estimation of $\theta_{\text {elbow }}$. This issue introduces serious disturbances to the morphing-wing controller that are difficult to reject and it also causes variations on the motion range of the elbow joint.

The column $(b)$ of Figure 6 shows the aerodynamic data corresponding to the applied wing kinematics from column (a). The aerodynamic data has been captured within a windtunnel using a force sensor attached to the body of the robot, as shown in Figure 5. The lift $\left(C_{L}\right)$ and $\operatorname{drag}\left(C_{D}\right)$ coefficients have been calculated from the aerodynamic force measurements in (6), as:

$$
\begin{aligned}
& C_{L}=2 L\left(\rho V_{\text {air }}^{2} A_{b}\right)^{-1} \\
& C_{D}=2 D\left(\rho V_{\text {air }}^{2} A_{b}\right)^{-1},
\end{aligned}
$$

with air density $\rho=1.2\left(\mathrm{Kgm}^{3}\right)$ and wing area $A_{b}=$ $0.05\left(\mathrm{~m}^{2}\right)$. Measuring 16 data points of $C_{L}$ and $C_{D}$ has taken one minute per angle of attack. In other words, the robot remained fixed at the corresponding angle of attack for one minute of continuous operation. Once the SMA wires were cooled again, the actuator raised the maximum operating frequency for another minute (fatigue issues). As it can be observed from the mid and bottom plots of the Figure 6(b), the lift coefficient decreases and the drag coefficient increases depending on the wing modulation pattern applied. In both cases, the upstroke portion of the wingbeat cycle takes longer (see mid and bottom plots of the Figure 6(a)), which means the wings generate more drag and less lift compared to the modulation pattern described by the top plot of the Figure 6(a).

The column (c) of Figure 6 shows how the net body forces (see $F_{n e t}$ in (7)) are also affected by changing the wing modulation patterns from column $(a)$. Comparing the bias of the net body forces between the mid and top plots of Figure 6(c), $F_{\text {net }}$ is increased by $28 \%$. Table 2 summarizes the numerical data. The experiments have confirmed that the net body forces decrease by applying wing modulation patterns with equal proportions of wing contraction-extension (mediumplots) or when the wing contraction during upstroke takes longer (bottom plots). However, despite the elbow joint motion is not periodic due to the SMA actuators, $F_{n e t}$ remains periodic because the flapping motion of the robot is periodic. Actually, the flapping motion (driven by the shoulder angle) is the only one that is periodic, as shown in the blue plot of Figure $5\left(\theta_{\text {flap }}\right)$. In free flight, the robot should pitch forward and backward following the natural oscillation produced by the wingbeat (downstroke/upstroke). This is why $F_{n e t}$ oscillates periodically.

In general, the wing modulation pattern depicted in the top plot of Figure 6(a) enables more performance in terms of lift, net body force generation and drag reduction than the other ones (medium and bottom plots). This is achieved thanks to the fact that the elbow joint contracts faster during upstroke and sufficiently to reduce the wing area at minimum span: on average, the upstroke should take about $37.5 \%$ of the wingbeat period. Despite aerodynamic loads are mostly produced in the upstroke due to drag forces, our goal is to apply a wing modulation pattern that reduces the energy consumption during the upstroke phase. The energy profiles can be observed in Figure 7. The top plots show the kinetic energy calculated with the model described in Section 3. Note that the columns (a), (b) and (c) correspond 
Table 2. Numerical data from experiments in Figures 6 and 7.

\begin{tabular}{lcccccccccc}
\hline Experiment & $\alpha(\mathrm{o})$ & $V_{\text {air }}\left(\mathrm{ms}^{-1}\right)$ & $f_{\text {flap }}^{-}(\mathrm{Hz})$ & $C_{L}$ & $C_{D}$ & $\bar{f}_{L}(\mathrm{~N})$ & $\bar{f}_{D}(N)$ & $f_{m g}(\mathrm{~N})$ & $F_{\text {net }}^{-}(\mathrm{N})$ & $K_{p e a k}(\mathrm{~J})$ \\
\hline Top & 10 & 5 & 2.25 & 1.5 & 0.152 & $1 . \overline{1} 2$ & $0 . \overline{1} 1$ & 0.77 & 0.115 & 0.0019 \\
Middle & 10 & 5 & 2.25 & 1.23 & 0.3 & $0 . \overline{9} 2$ & $0 . \overline{2} 2$ & 0.77 & 0.09 & 0.0018 \\
Bottom & 10 & 5 & 2.25 & 0.48 & 0.17 & $0 . \overline{3} 6$ & $0 . \overline{1} 2$ & 0.77 & 0.021 & 0.0013 \\
\hline
\end{tabular}
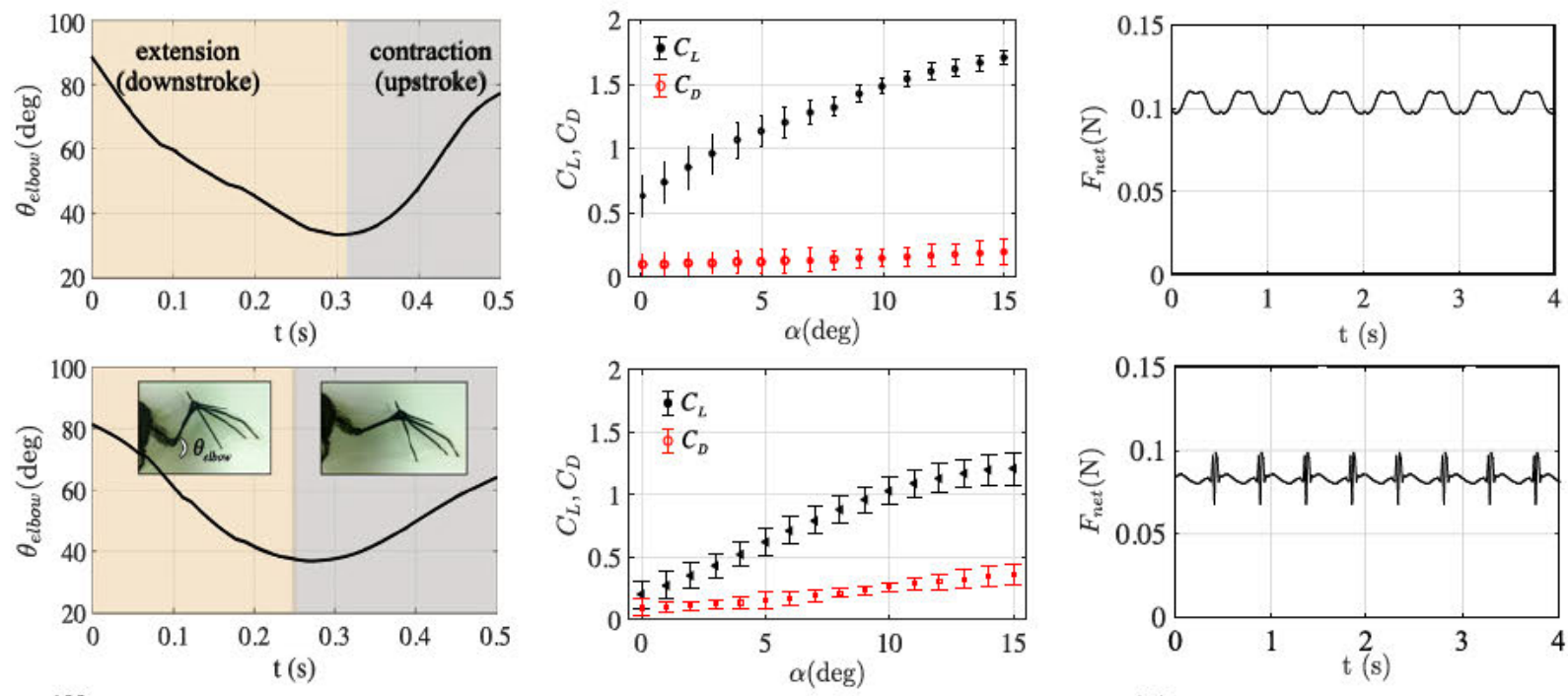

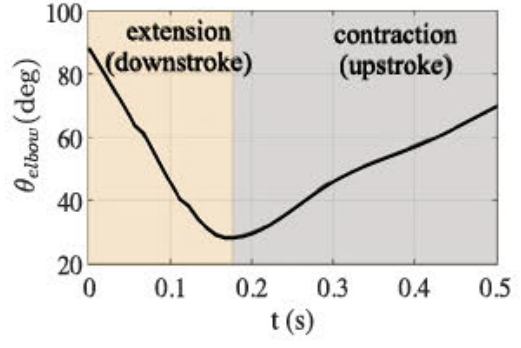

(a)

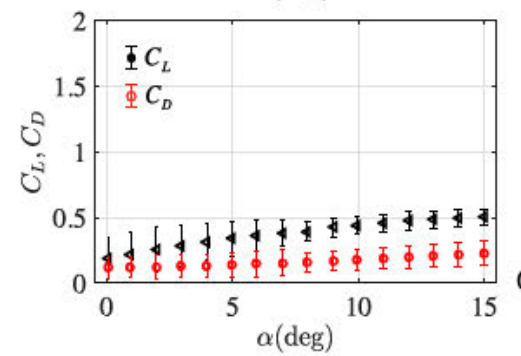

(b)

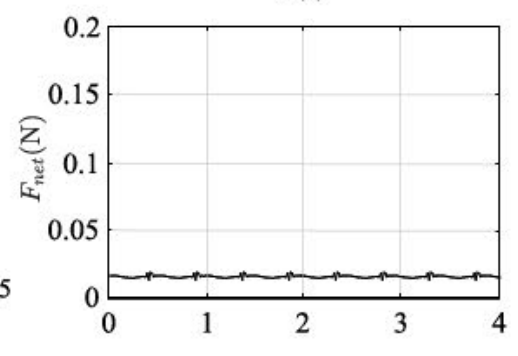

(c)

Figure 6. (Experimental) influence of applying different wing modulation patterns on lift, drag, and net body force generation. Airspeed in the wind tunnel $V_{\text {air }}=5 \mathrm{~ms}^{-1}$, wingbeat frequency $f_{\text {flap }}=2 \mathrm{~Hz}$, wingstroke amplitude (flapping) $\theta_{\text {flap }}=120^{\circ}:(a)$ close-up to a wingbeat cycle for a set of wing modulation patterns driven by $\theta_{\text {elbow }}$. The wing area changes from 0.05 to $0.0385 \mathrm{~m}^{2}$ when wings are fully retracted. $(b)$ The impact of changing the wing modulation patterns on: the lift coefficient $C_{L}$ and drag coefficient $C_{D}$ as a function of the angle of attack $\alpha$, and (c) net body force generation $F_{\text {net }}$ measured at the center of mass of the robot. Net forces have been measured at $\alpha=10^{\circ}$.

to the kinetic energy for the experiments shown in Figure 6 respectively.

The bottom plots of Figure 7 show a close-up to a wingbeat cycle. Unlike the energy cost profiles shown in the columns (b) and (c), the energy cost profile shown in the column (a) is homogeneously distributed during the entire wingbeat. As demonstrated by [9], the energy cost measured in real bats is approximately homogeneously over the course of a wingbeat cycle. Also, the energy cost is lower during the upstroke phase than the one required during the downstroke. This result might prove that a faster wing contraction might enable the robot to counteract aerodynamic load effects (mostly produced in the upstroke).

In conclusion, the reason about why the wing modulation patterns shown by the mid and bottom plots of Figure 6(a) display such a poor performance in terms of lift generation, drag reduction and net body force generation (cf. Figure $6(\mathrm{~b})$ and (c)) is clearly observed in the energy cost profiles of Figures 7(b) and (c). During flapping, the upward and backward accelerations of the wings produce an inertial force that will move the body forward and downward with respect to the wingstroke. The upstroke phase is crucial 

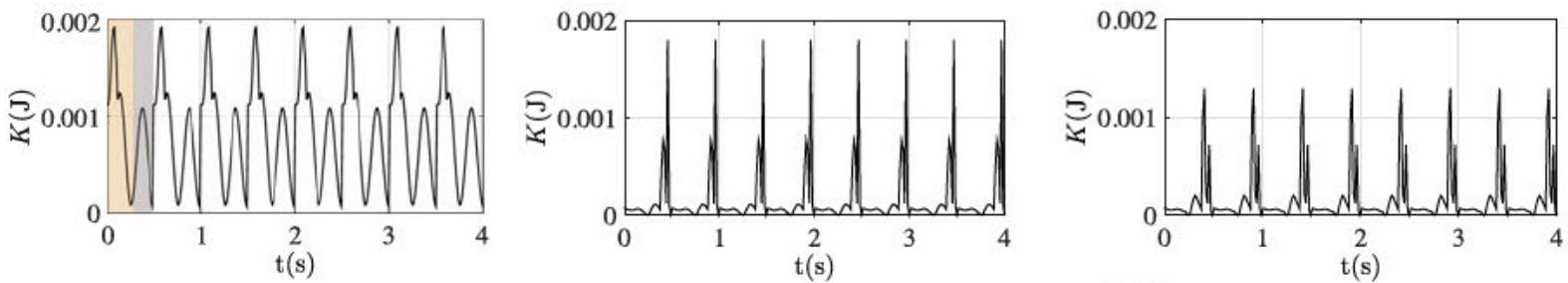

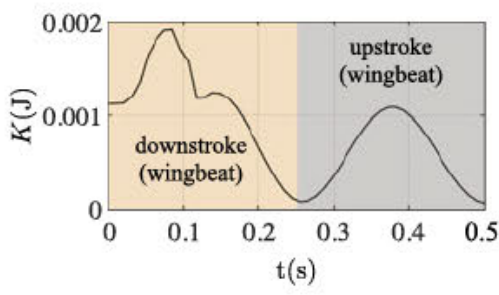

(a)

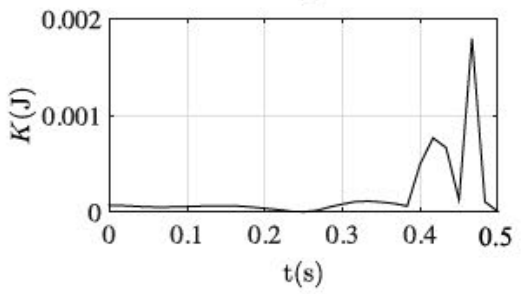

(b)

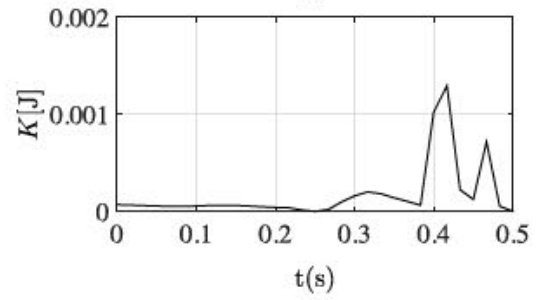

(c)

Figure 7. Energy cost profiles obtained from the applied wing modulation patters from 6(a). The plots in the bottom close up the energy cost during one wingbeat cycle: downstroke and upstroke. The kinetic energy has been measured at $\alpha=10^{\circ}$.

because mostly of the thrust that is essential to drive the robot forward is generated during the upstroke, whereas lift forces are mostly generated during the downstroke. Note that during the downstroke phase, the energy cost is low because despite that both wings extend faster, low spatial body velocities $\left(V_{b}\right)$ are produced: in free-flight, the robot's body should accelerate forward during upstroke, not during the downstroke $[13,20]$. Also, by extending the wings faster during the downstroke, the lifting area of the wing profile is reduced from 0.05 to $0.0385 \mathrm{~m}^{2}$, which in turn causes poor lift generation.

\section{Conclusions}

In this work, we were inspired by the unmatched flying skills of bats. Based on the biological studies cited earlier, our working hypothesis of improving lift, net body force generation and energy cost was achieved by the proper control of the wing's geometry. To test such hypothesis and provide a quantification of the above described effects, we have studied how different wing modulation patterns might affect the overall performance of BaTboT's wing movements. We have found that in our robotic model, the contraction time of the wings during the upstroke should be faster than the extension time during the downstroke; on average, the upstroke should take about $37.5 \%$ of the wingbeat period. This enables to reduce the drag coefficient from 0.3 to $0.152(50 \%)$ and to drastically increase the lift coefficient from 0.48 to 1.5 . Also, net body forces are increased by $28 \%$ (from 0.09 to $0.115 \mathrm{~N}$ ) at the expense of an acceptable energy cost peak of $0.0019 \mathrm{~J}$. The upstroke phase of the wings is crucial because during that period of time the downward motion of the wings makes the center of mass of the robot to accelerate forward as long as drag forces are low.
By now, the SMA bandwidth limitation does not allow to increase the wingbeat frequency higher than $2.5 \mathrm{~Hz}$. Therefore, the results presented in this paper cannot be completely generalized or linearly scaled to higher frequencies, but the experiments show positive insights on how performance is behaving (lift, drag, $F_{n} e t$, energy), at least at lower frequencies.

The three scenarios depicted in Figure 6 reflect the differences of applying slower, faster or equal periods for wing contraction-extension during downstroke and upstroke. Unfortunately, SMA inaccuracy in terms of position tracking and actuation speed (see Figure A3) does not allow to perfectly quantified which is the exact elbow pattern that maximizes lift and reduces energy consumption. To solve this, an optimization approach must be addressed, in which the wing profiles result as the output of minimizing an energy cost function. Upcoming work will be focus on how to optimize the performance of the robot and to improve the accuracy of the SMA actuators. It is also necessary to investigate high-frequency response of the SMAs and the possibility of using high-bandwidth force control as a possible approach to enable higher wingbeat frequencies and remove fatigue issues.

Also, we need to increase lift and net body forces to clearly counteract the robot's weight. In fact, real bats with a mass between 100 and $200 \mathrm{~g}$ flap between 5 and $15 \mathrm{~Hz}$ depending on the maneuver, and generate an average lift coefficient of around 1.0, as shown in the figure 5 from [10]. It seems like we are not too far away from those numbers; however, most of the amazing dexterity, maneuverability, and efficiency presented by real bats is due to their bio-mechanics: body control, hundreds of muscles, their sensing apparatus, etc. We expect that some of the results obtained with the tethered robot, give us a clear 
vision about the path that we need to follow on pursuing free flight.

\section{Acknowledgements}

The authors would like to thank to professors K. Breuer and S. Swartz from Brown University for providing the support and useful knowledge about the robot design, bat flight kinematics, and aerodynamics. To Joe Balhman for his contribution on the original design of the highly articulated wing skeleton used by BaTboT.[18,19]

\section{Notes on contributors}

Julian Colorado is an assistant professor in the Department of Electronics Engineer- ing at Pontificia Universidad Javeriana. He completed his $\mathrm{PhD}$ and MS in Robotics at Universidad Politecnica de Madrid, Spain, where he studied the development of novel flight controllers (GNC) for a diverse category of Unmanned Aerial Vehicles, including quad- rotors and highly-articulated morphing wing drones inspired by the biomechanics of bats. He was a visiting research fellow at Brown University, USA (2010-2011), where he studied how to integrate smart-actuators based on Shape Memory Alloys to control wing modulation in a bat-like robot. Julian Colorado research interests include Field Robotics, Aerial Robotics, Bio-inspired robotics, and Guidance Navigation Control -GNC.

Claudio Rossi received his $\mathrm{PhD}$ in Computer Science from the University of Bologna, Italy, in 2001. He has been visiting researcher at the LIACS (Leiden Institute of Advanced Computer Science, NL), post-doctoral fellow at the Department of Artificial Intelligence at the UPM and visiting professor at the University Carlos III of Madrid. During 2005-09 he was 'Ramon y Cajal' research fellow at the UPM, and since 2009 he is professor at the UPM. His research interests, initially centered on Nature-inspired search techniques for hard optimization problems, have been later directed towards applications of Artificial Intelligence to robotics. Since 2009, his research interests have been directed towards and bio-inspired systems, shifting form Nature-based (software) search techniques to Nature-based physical systems. He has participated in several international research projects, and he has published around 80 papers in scientific journals and international conferences, on the topics of artificial intelligence and robotics.

Chao Zhang received the BSc degree in detection homing and control technology in 2010 and the MSc degree in system engineering in 2013 from the Northwestern Polytechnical University, Xi'an, China. Now he is currently working toward the $\mathrm{PhD}$ degree in automatic control and robotics from the Universidad Politecnica de Madrid, Madrid, Spain. His research interests include kinematics and dynamics of robots, flapping-wing MAVs, compliant mechanisms and optimization design.
Antonio Barrientos received the Msc Engineer degree by Automatic and Electronic from the Polytechnic University of Madrid in 1982, and the $\mathrm{PhD}$ in Robotics by the same University in 1986. In 2002, he obtained de MSc Degree in Biomedical Engineering by Universidad Nacional de Educación a Distancia. Since 1988 he is Professor on robotics, computers, and control engineering at the Polytechnic University of Madrid. He has worked for more than 30 years in robotics, developing industrial, and service robots for different areas. Currently his main interests are field robotics, with special applications to agriculture and security and defence. He is author of several textbooks in Robotics and Manufacturing automation. He is also co-author of more than 100 scientific papers in journals and conferences. He is Editorial Board Member and reviewer of several journals in Robotics and Control engineering. Also he is a senior member of the IEEE. Antonio Barrientos is currently the head of the Robotics and Cybernetics research group of the Centre for Automatic and Robotic in the Technical University of Madrid - -Spanish National Research Council.

\section{References}

[1] Hedenstrom A, Johansson L, Spedding G. Bird or bat: comparing airframe design and flight performance. Bioinspir. Biomim. 2009;4:5001-5013.

[2] Gomez J, Garcia E. Morphing unmanned aerial vehicles. Smart Mater. Struct. 2011;20:1-16.

[3] Winter Y, Helversen OV. The energy cost of flight: do small bats fly more cheaply than birds? J. Comp. Physiol. B. 1998;168:105-111.

[4] Daniel K, Riskin K, Bergou A, Breuer K, Swartz S. Stroke wing flexion and the inertial cost of bat flight. Proc. R. Soc. B. 2012;279:2945-2950.

[5] Lentink D, Biewener A. Nature inspired flight-beyond the leap. Bioinspir. Biomim. 2010;5:040201.

[6] Lentink D, Muller U, Stamhuis E, et al. How swifts control their glide performance with morphing wings. Nat. Lett. 2007;446:1082-1085.

[7] Swartz S, Iriarte J , Riskin D, Breuer K . A bird? A plane? No, it's a bat: an introduction to the biomechanics of bat flight. In: Evolutionary history of bats fossils, molecules and morphology. Edited by Gregg F. Gunnell and Nancy B. Simmons . Cambridge University Press; 2012. p. 317-352.

[8] Thollesson M, Norberg U. Moments of inertia of bat wings and body. J. Exp. Biol. 1991;158:19-35.

[9] Riskin D, Bergou A, Breuer K, Swartz S. Upstroke wing flexion and the inertial cost of bat flight. Proc. R. Soc. B: Biol. Sci. 2012;279:2945-2950.

[10] Riskin D, Iriarte-Diaz J, Middleton K, Breuer K, Swartz S. The effect of body size on the wing movements of pteropodid bats, with insights into thrust and lift production. J. Exp. Biol. 2010;213:4110-4122.

[11] Tian X, Iriarte-Diaz J, Middleton K, et al. Direct measurements of the kinematics and dynamics of bat flight. Bioinspir. Biomim. 2006;1:S10-S18.

[12] Riskin D, Willis D, Iriarte-Diaz J, et al. Quantifying the complexity of bat wing kinematics. J. Theor. Biol. 2008;254:604-615.

[13] Iriarte-Diaz J, Riskin D, Willis D, Breuer K, Swartz S. Whole-body kinematics of a fruit bat reveal the influence of wing inertia on body accelerations. J. Exp. Biol. 2011;214:1546-1553. 
[14] Bunget G. A bio-inspired micro-aerial vehicle for flapping flight [Ph.D. thesis]. North Carolina State University; Raleigh 2010.

[15] Yang S, Seelecke S. Fe analysis of sma-based bio-inspired bone joint system. Smart Mater. Struct. 2009;18:104020 104030.

[16] Callahan R, Garcia E. Bio-inspired bat wing design and fabrication. Ithaca (NY): Cornell university; 2010.

[17] Leylek EA, Manzo JE, Garcia E. Bat-inspired wing aerodynamics and optimization. J. Aircr. 2010;47:323-328.

[18] Bahlman JW, Swartz SM, Breuer KS. Design and characterization of a multi-articulated robotic bat wing. Bioinspiration Biomimetrics. 2013;8:016009.

[19] Colorado J, Barrientos A, Rossi C, Bahlman JW, Breuer KS. Biomechanics of smart wings in a bat robot: morphing wings using sma actuators. Bioinspiration Biomimetrics. 2012;7:036006.

[20] Colorado J, Barrientos A, Rossi C, Parra C. Inertial attitude control of a bat-like morphing-wing air vehicle. Bioinspir. Biomim. 2013;8:016001.

[21] Featherstone R. Rigid body dynamics algorithms. New York (NY): Springer; 2008.

[22] Jbac A. A unified formulation for massively parallel rigid multibody dynamics of o( $\log 2 n)$ computational complexity. J. Parallel Distrib. Comput. 2002;62:1001-1020.

[23] Denavit J, Hartenberg R. A kinematic notation for lower pair mechanisms based on matrices. Trans. ASME J. Appl. Mech. 1955;22:215-221.

[24] Sm S, Md G, Hd K, Wr W. Mechanical properties of bat wing membrane skin. J. Zool. 1996;239:357-78.

[25] Elahinia M, Ahmadian M. An enhanced sma phenomenological model: I. The shortcomings of the existing models. Smart Mater. Struct. 2005;14:1-13.

[26] Teh Y, Featherstone R. An architecture for fast and accurate control of shape memory alloy actuators. Int. J. Rob. Res. 2008;27:595-611.

[27] GSong N, Lee H. Position control of shape memory alloy actuators with internal electrical resistance feedback using neural networks. Smart Mater. Struct. 2004;13:777-783.

\section{Appendix 1. Morphing-wings driven by SMA muscles}

Shape memory alloy actuators acting as biceps and triceps artificial muscles are connected to the elbow joint, providing direct actuation over $\theta_{\text {elbow }}$ and enabling an elbow rotation range of $60^{\circ}$. The Migamotor actuator consists of six wires of SMA with $150 \mathrm{mu}$ of thickness which are attached to metal strips stacked in parallel. Each SMA strip pulls when heated to make a stroke of $4 \mathrm{~mm}$. SMA actuators provide powerful linear actuation to control the wing modulation by means of reproducing the functioning of the biceps and triceps muscles. Basically, SMA wires contract upon the heating that is produced by an electrical current and then recover the original length when cooled. This is known as the shape memory effect. The more input power is applied to SMA, the faster will contract, but the lower will extend. Besides, overstressing the NiTi wires might cause physical damage to the shape memory effect.[26]
Figure A1(a) details the experimental setup for SMA dynamic characterization based on the frequency response methodology presented in [26]. In general, the applied heating power $u_{\text {heating }}=$ $a+b \sin (2 \pi f t)$ is converted to a current signal using the nominal value for the SMA wire electrical resistance provided by the manufacturer $\left(R_{s m a}=8.5 \Omega\right)$. The output force $(F)$ is measured using a sensor with 0.318 gram-force of resolution and then mapped to a torque value corresponding to the elbow joint torque. Note in Figure A1(a) that an antagonistic configuration composed by two SMA actuators are required for actively controlling the direction of the elbow joint rotation. Each SMA actuator produces an output pull-force that subsequently generates a torque acting on the elbow and wrist joints ( $\tau_{\text {elbow }}$ ).

The magnitude and phase of the sine wave component of the signal $F$ are measured over a frequency range from 0.1 to $100 \mathrm{~Hz}$ in order to characterize the SMA actuation. A least-squares methodology is used for extracting the sine wave component in the recorded force data $(F)$. Figure A1(b) shows that the SMA frequency response is similar of a first-order linear system, where the frequency at which the change of slope in magnitude occurs is known as a pole $(s=-2.857)$. Several measurements have indicated that the suitable transfer function that fits the experimental data are:

$$
\tau_{\text {elbow }}=0.016(0.35 s+1)^{-1} u_{\text {heating }}
$$

The first-order linear system in (A1) is validated by comparing its time-response against the experimental data measured using the force sensor. That comparison is shown in the bottom plot of Figure A1(b). The measured output torque was generated by applying an input power of the form: $u_{\text {heating }}=a+b \sin (2 \pi f t)$ with a DC bias of the input power of $a=3.06 \mathrm{~W}$, an small-signal amplitude of $b=2 \mathrm{~mW}$ and a driven frequency of $f=2 \mathrm{~Hz}$. The applied input power corresponds to an input current of $I_{\mathrm{sma}}=600 \mathrm{~mA}$. Table A1 reports operation data of the SMA actuators.

Once the model in (A1) is validated, we propose a control architecture for regulating the wing shape (see Figure A2). The control strategy is driven by a Proportional-Integral-Derivative (PID) controller of the form: $u_{\text {heating }}(s)=K_{p}+K_{i} s^{-1}+K_{d} s$ with parameters tuned based on the actuation dynamic model from (A1). Such parameters are $K_{p}=35, K_{i}=0.006$ and $K_{d}=0.08$.

In Figure A2, the module called morphing-wing joint trajectories generates modulation patterns for both shoulder and elbow joints. The shoulder reference is mapped to a DC motor that drives the primary flapping motion whereas the elbow reference is an input to the PID controller. The position error is determined by comparing the elbow reference against the feedback of the elbow joint position, which in turn is estimated by means of measuring the electrical resistance of the SMA wires $\left(R_{S m a}\right)$ within the control loop. SMA can be used not only as actuators, but also as sensors [27]. The electrical resistance of the SMA wires $\left(R_{s m a}\right)$ changes linearly with the SMA strain that is kinematically coupled to the rotation of the elbow joint $\left(\theta_{\text {elbow }}\right)$. Further details about the morphing-wing controller can be found in [19]. 
Table A1. SMA actuation data.

\begin{tabular}{lc}
\hline Parameter & Value \\
\hline Wing contraction speed $\dot{\theta}_{\text {elbow }}[\mathrm{deg} / \mathrm{s}]$ & $\sim 300^{\mathrm{a}}$ \\
Input heating power $P[\mathrm{~W}]$ & 3.06 \\
Input heating current $I_{\text {sma }}[\mathrm{mA}]$ & 600 \\
Output torque $\tau_{\text {elbow }}[\mathrm{Nm}]$ & 0.02 \\
\hline
\end{tabular}

The elbow rotates $60^{\circ}$ in $200 \mathrm{~ms}$

(a)

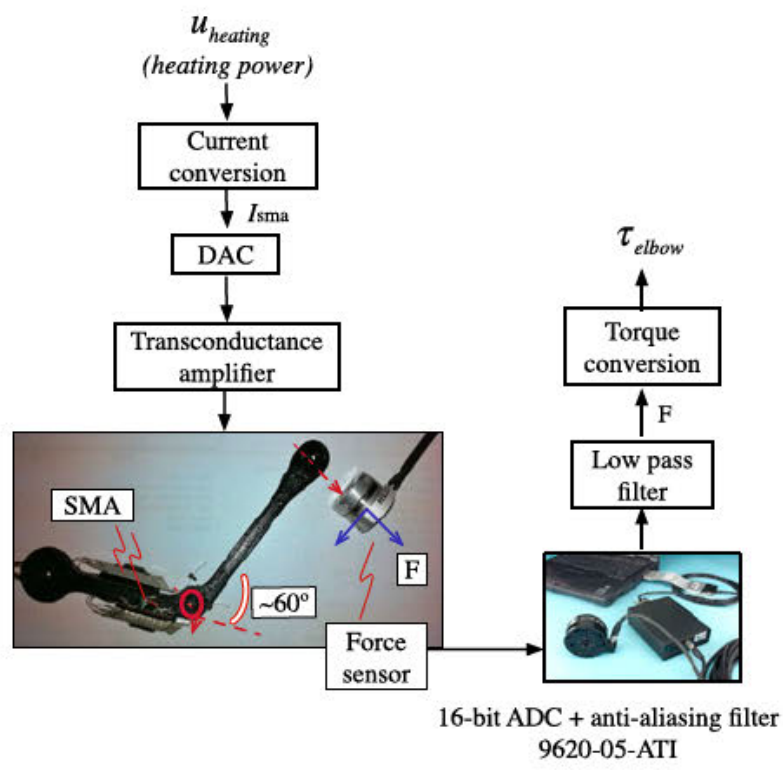

(b)

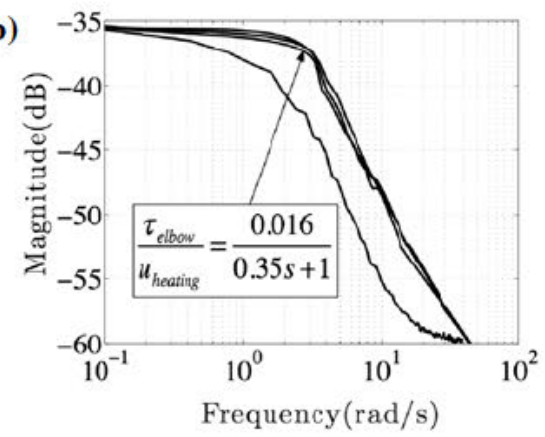

(c)

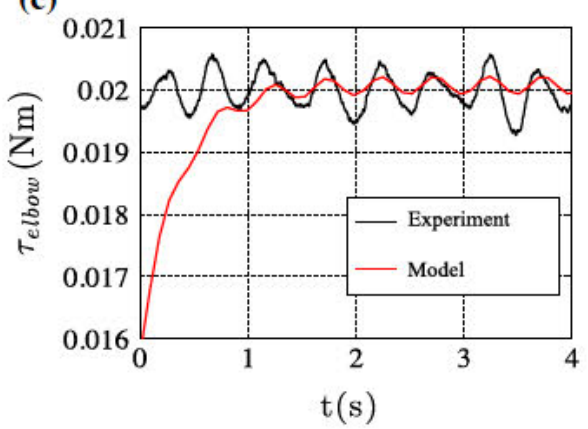

Figure A1. (a) Setup for SMA dynamic characterization, (b) SMA dynamic characterization based on the frequency response methodology presented in [26], (c) output-torque is measured by applying small-signal power of the form: $u_{\text {heating }}=a+b \sin (2 \pi f t)$ with $f=2 \mathrm{~Hz}$.

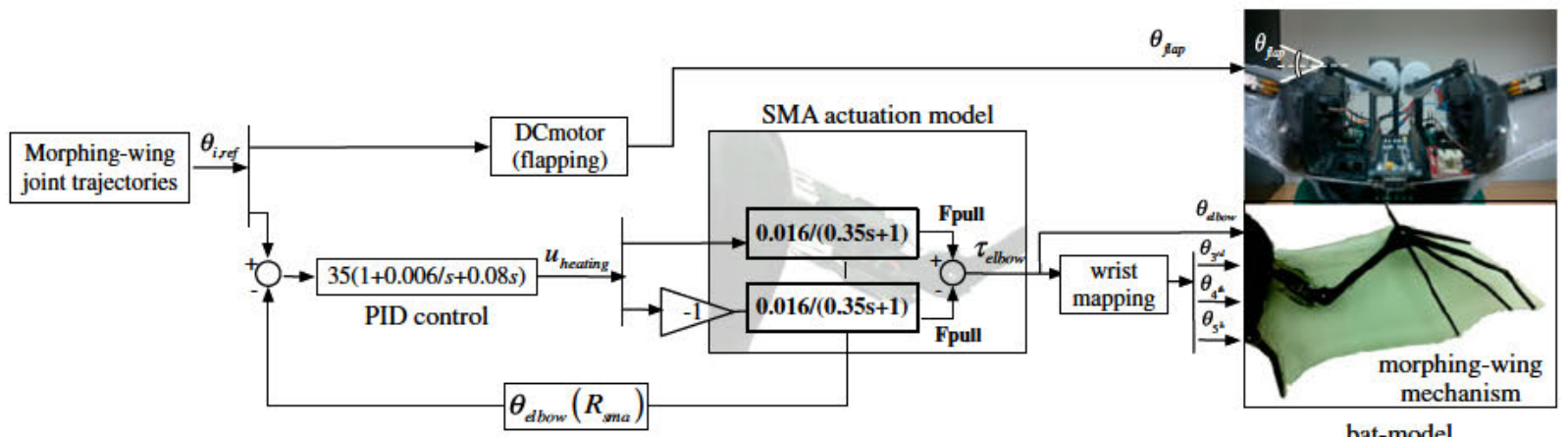

Figure A2. Morphing-wing control architecture. 

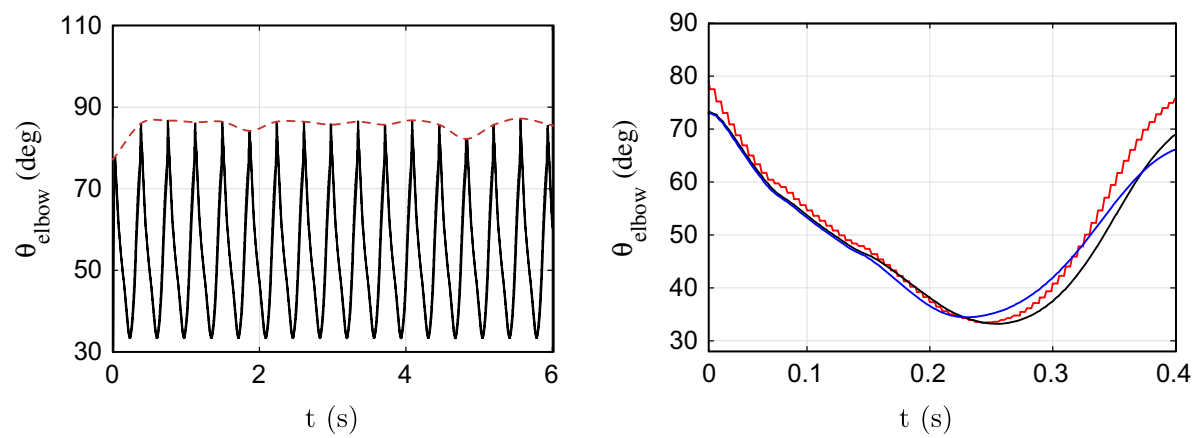

Figure A3. (Left) elbow joint trajectory profile at $f=2.5 \mathrm{~Hz}$, the dashed line highlights how the amplitude changes during each wingbeat cycle. (Right) close-up to a wingbeat cycle: a set of elbow joint profiles depending on SMA actuation uncertainties. 